\title{
Long-term validation of a molecular progression-associated gene classifier for prediction of muscle invasion in primary non-muscle-invasive bladder cancer
}

\author{
HO WON KANG ${ }^{1}$, SUNG PIL SEO ${ }^{1}$, PILDU JEONG ${ }^{1}$, YUN-SOK HA ${ }^{2}$, WON TAE KIM ${ }^{1}$, \\ YONG-JUNE KIM ${ }^{1}$, SANG-CHEOL LEE ${ }^{1}$, SEOK-JOONG YUN ${ }^{1}$ and WUN-JAE KIM ${ }^{1}$ \\ ${ }^{1}$ Department of Urology, Chungbuk National University Hospital, Chungbuk National University, College of \\ Medicine and Institute for Tumor Research, Cheongju, Chungbuk 28644; ${ }^{2}$ Department of Urology, School \\ of Medicine, Kyungpook National University Medical Center, Daegu 41404, Republic of Korea
}

Received October 2, 2016; Accepted March 21, 2017

DOI: $10.3892 / \mathrm{ol} .2017 .6408$

\begin{abstract}
Our previous study reported a clinically applicable prognostic gene classifier for primary non-muscle-invasive bladder cancer (NMIBC). The present study aimed to perform long-term validation of this classifier in the prediction of muscle-invasive disease. Previously published gene expression profiles were used from 176 patients with NMIBC with extended follow-up. Progression was defined as development of muscle invasion or metastasis, and the progression risk score was calculated using the previously developed eight-gene progression classifier. During median follow-up of 72.8 (interquartile range, 37.0-118.7) months, 26 (14.8\%) patients progressed to muscle-invasive bladder cancer. The molecular progression risk score was significantly associated with clinicopathological variables, including tumor number, stage, grade and multivariate risk assessment tools $(\mathrm{P}<0.05$ in each case). Multivariate Cox regression analysis revealed that molecular progression risk score was an independent predictor of development of invasive tumor, either as a continuous variable [hazard ratio (HR), 1.489; 95\% confidence interval (CI), 1.216-1.823; $\mathrm{P}<0.001]$ or as a categorical variable (HR, 5.026; 95\% CI, 1.619-15.608; $\mathrm{P}=0.005)$. In conclusion, the present results confirmed the clinical utility of the progression-associated gene classifier for prediction of development of muscle invasion in NMIBC. The molecular progression risk score
\end{abstract}

Correspondence to: Professor Seok-Joong Yun or Professor Wun-Jae Kim, Department of Urology, Chungbuk National University Hospital, Chungbuk National University, College of Medicine and Institute for Tumor Research, 776 1sunhwan-ro, Seowon-gu, Cheongju, Chungbuk 28644, Republic of Korea

E-mail: sjyun@chungbuk.ac.kr

E-mail:wjkim@chungbuk.ac.kr

Key words: bladder cancer, gene signature, progression, risk score may aid in selecting patients who could benefit from more aggressive therapeutic intervention.

\section{Introduction}

Bladder cancers (BCs) are mixtures of heterogeneous cell populations, and multiple factors are involved in determining their recurrence, progression and survival (1). Whereas the overall survival rate of patients with non-muscle-invasive bladder cancer (NMIBC) is excellent compared with that in other malignancies, several of these patients exhibit a high risk of recurrence and a variable risk of progression despite administration of local therapies $(2,3)$. A total of $10-20 \%$ of patients with NMIBCs subsequently develop invasive or metastatic cancer $(4,5)$. Muscle-invasive BC (MIBC) is often a life-threatening disease with short curable period, and the oft-cited $50 \%$ overall survival at 5 years for MIBC has remained relatively unchanged in 20 years $(6,7)$. Accordingly, identifying patients at risk of developing MIBC is essential for appropriate disease management in patients with NMIBC. Clinical risk factors for progression include: Invasion of the lamina propria, high grade based on the World Health Organization/International Society of Urologic Pathology consensus classification $(8,9)$, tumor size, occurrence of carcinoma in situ (CIS) and multiplicity or recurrence of high-risk tumors (10-12). Two multivariate risk assessment tools for predicting the outcome of NMIBC have been developed $(13,14)$. However, currently none of the predictive tools based on conventional clinical and pathological parameters are sufficiently sensitive or specific to detect, monitor and determine the prognosis of BC (15). To overcome these limitations, studies have focused on identifying molecular markers that enable clinicians to classify BCs in more detail, thereby enabling selection of the optimal treatment regimen $(16,17)$.

Previously, microarray technology has facilitated the development of numerous cancer classifiers, identification of tumor subclasses, discovery of progression markers and prediction of disease outcome in numerous types of cancer (18). Molecular staging may provide increased accurate predictions of patient outcome compared with the currently employed 
histopathological staging, and may also improve treatment outcomes by enabling treatment to be tailored to the severity of the disease (19). Our previous study performed a microarray analysis of specimens derived from 103 primary NMIBCs and identified an eight-gene progression-associated gene classifier (Table I) (20). The original study defined progression as TNM upstaging, and included several cases of progression between Ta and T1. In the present study, our previously published progression-associated gene classifier (20) was validated in the prediction of muscle invasion in NMIBC over an extended follow-up period.

\section{Materials and methods}

Patients and tissue samples. The present study was performed in agreement with applicable laws and regulations, good clinical practices and ethical principles as described in the Declaration of Helsinki. The Ethics Committee of Chungbuk National University (Cheongju, Korea) approved this protocol (IRB approval no. 2010-01-001), and written informed consent was obtained from each patient. Collection and analysis of all samples was approved by the Institutional Review Board of Chungbuk National University.

The present study utilized previously published gene expression profiles from 176 consecutive primary NMIBC tumor specimens with histologically verified transitional cell carcinoma. The patients from whom these tumors were derived underwent transurethral resection (TUR) at Chungbuk National University Hospital in South Korea between January 1995 and December 2009. To make the study population more homogeneous, patients with concomitant CIS or who had undergone radical cystectomy were excluded. To avoid the risk of under-staging, or when a high-grade tumor was detected, a second TUR was performed 2-4 weeks after initial resection if the original BC specimen did not include proper muscle tissue. Patients with a T1 tumor, multiple tumors, large tumors ( $\geq 3 \mathrm{~cm}$ in diameter) or a high-grade tumor received one cycle of intravesical Bacillus Calmette-Guerin (BCG) treatment. Following initial TUR, each patient was monitored according to standard guidelines (21). Response to treatment was assessed by cystoscopy and urinary cytology. Patients who were disease-free within 3 months subsequent to treatment were assessed every 3 months for the first 2 years and subsequently every 6 months. Tumors were staged and graded according to the 2002 tumor-node-metastasis (TNM) classification and the 1973 World Health Organization grading system $(22,23)$. A follow-up period of $\geq 6$ months was required (unless recurrence and/or progression occurred within 6 months). Progression was defined as muscular invasion or metastatic disease. The clinical and pathological progression risk was calculated using two multivariate risk assessment tools, the European Organization for Research and Treatment of Cancer (EORTC) risk tables and the Spanish Urological Club for Oncological Treatment (CUETO) prognostic scoring model $(13,14)$.

All tumors were macrodissected within 15 min of surgical resection, fresh-frozen in liquid nitrogen and stored at $-80^{\circ} \mathrm{C}$ until use. Each cancer specimen was confirmed as representative by pathological confirmation of adjacent tissue in fresh-frozen sections from TUR specimens. Resected tumors were fixed in $4 \%$ paraformaldehyde in $0.1 \mathrm{M}$ sodium phosphate buffer for $24 \mathrm{~h}$ at room temperature. The sections were processed on a computerized tissue processor (Tissue-Tek VIP; Sakura Finetek USA, Inc., Torrance, CA, USA) and embedded in paraffin (Paraplast Medium; Leica Biosystems, Wetzlar, Germany) on a tissue embedding console system (Tissue-Tek TEC; Sakura Finetek USA, Inc.). All paraffin embedded tissue blocks were sectioned at $4 \mu \mathrm{m}$ thickness with a microtome, and slides were then prepared. Deparaffinization and rehydration of the sections was performed using 4 incubation steps of xylene and a series of $100 \%$ ethanol, $95 \%$ ethanol and twice with $70 \%$ ethanol. Each step was performed for $5 \mathrm{~min}$ at room temperature. The sections were deionized using water for $15 \mathrm{~min}$ at room temperature. Subsequently, the sections were stained with hematoxylin for $5 \mathrm{~min}$ and eosin for $10 \mathrm{sec}$ at room temperature. Final stages of processing were performed with an automated slide stainer (Tissue-Tek Prisma; Sakura Finetek USA, Inc.) and automated coverslip (Tissue-Tek Glas; Sakura Finetek USA, Inc.). Sections were examined under a light microscope under magnifications, x100 and x400 (Olympus BX53; Olympus Corp., Tokyo, Japan).

Selection of prognosis-associated gene classifiers using microarray gene expression profiling. The experimental and statistical method for determining the eight-gene progression-associated classifier was described previously (20). The full microarray data set is available online (http://www.ncbi. nlm.nih.gov/geo/) under accession number GSE13507.

RNA extraction and construction of cDNA. RNA was isolated from tissue using $1 \mathrm{ml}$ TRIzol reagent (Invitrogen; Thermo Fisher Scientific, Inc., Waltham, MA, USA) and homogenized in a $5 \mathrm{ml}$ glass tube. The homogenate was then transferred to a $1.5 \mathrm{ml}$ tube and mixed with $200 \mu \mathrm{l}$ chloroform. Following $5 \mathrm{~min}$ incubation at $4^{\circ} \mathrm{C}$, the homogenate was centrifuged for $13 \mathrm{~min}$ at $13,000 \mathrm{xg}$ at $4^{\circ} \mathrm{C}$. The upper aqueous phase was transferred to a clean tube and $500 \mu \mathrm{l}$ isopropanol was added. The mixture was incubated for $60 \mathrm{~min}$ at $4^{\circ} \mathrm{C}$, and the tube was centrifuged for $8 \mathrm{~min}$ at $13,000 \mathrm{x} \mathrm{g}$ at $4^{\circ} \mathrm{C}$. The upper aqueous phase was then mixed with $500 \mu 175 \%$ ethanol, and centrifuged for $5 \mathrm{~min}$ at $13,000 \mathrm{x}$ g at $4^{\circ} \mathrm{C}$. After discarding the upper aqueous layer, the pellet was dried at room temperature, dissolved in diethylpyrocarbonate-treated water, and then stored at $-80^{\circ} \mathrm{C}$. The quality and integrity of the RNA were confirmed by agarose gel electrophoresis and ethidium bromide staining, followed by visual inspection under ultraviolet light. cDNA was prepared from $1 \mu \mathrm{g}$ total RNA using a First-Strand cDNA Synthesis kit (Amersham; GE Healthcare, Chicago, IL, USA) according to the manufacturer's protocol.

Reverse transcription-quantitative polymerase chain reaction $(R T-q P C R)$. qPCR amplification was performed using a Rotor-Gene 6000 instrument (Corbett Life Science; Qiagen, Inc., Valencia, CA, USA) to quantify gene expression. qPCR assays were carried out in micro-reaction tubes (Corbett Life Science; Qiagen, Inc.) using SYBR-Premix Ex Taq (Takara Biotechnology Co., Ltd., Dalian, China). The primers used in the amplification are listed in Table I. The PCR reaction was performed in a final volume of $10 \mu \mathrm{l}$ consisting of $5 \mu \mathrm{l}$ 2X SYBR-Premix Ex Taq buffer, $0.5 \mu \mathrm{l}$ each sense and antisense primers $(10 \mathrm{pmol} / \mu \mathrm{l})$ and $1 \mu \mathrm{l} \mathrm{cDNA}$. The product 
Table I. Eight-gene progression-associated molecular classifiers for non-muscle-invasive bladder cancer.

\begin{tabular}{lcccc}
\hline Gene symbol & Sense primer (5'-3') & Antisense primer (5'-3') & $\begin{array}{c}\text { Cox regression } \\
\text { coefficients }\end{array}$ \\
\hline COCH & AGA AAG CAG ATG TCC TCT GC & TCC CCC TGA GTT GCT GAT TA & 1.190 & 0.173953307 \\
CELSR3 & CTC CAT GTT GGT GAC TGT CAC & TCC TGC CAC ATG TTC TCA AG & 1.246 & 0.21993842 \\
$H M O X 1$ & AAC TTT CAG AAG GGC CAG GT & CTT GTT GCG CTC AAT CTC CT & 1.251 & 0.223943231 \\
KIF 1 A & AAG AAC AAG GGC AAC CTT CG & CTC CAT TCA TGT TGG TGG CC & 1.060 & 0.058268908 \\
$M G C 17624$ & GTC CTG AAC GAC AAG CAC CT & AGG CTT CTG GGT CGA TTT CT & 0.670 & -0.400477567 \\
$M T A P$ & TCC TTG AGG GAG GAG ATT CA & TCC TCT GGC ACA AGA ATG AC & 0.906 & -0.098715973 \\
$P F K B 4$ & ACT GAA CCC CCT GAA GAA GA & ATG AGA GTT GGG CAG TTG GT & 1.400 & 0.336472237 \\
$S 100 A 8$ & CAT CGA CGT CTA CCA CAA GT & GAA TGA GGA ACT CCT GGA AG & 1.176 & 0.162118849 \\
\hline
\end{tabular}

$\mathrm{COCH}$, coagulation factor C homolog; CELSR3, EGF LAG seven-pass G-type receptor 3; HMOX1, heme oxygenase 1; KIF1A, kinesin family member 1A; MGC17624, chromosome 16 open reading frame 74; MTAP, methylthioadenosine phosphorylase; PFKFB4, 6-phosphofructo-2-kinase/fructose-2,6-biphosphatase 4; S100A8, S100 calcium binding protein A8.

was purified with a QIAquick Extraction kit (Corbett Life Science; Qiagen, Inc.), quantified with a spectrophotometer (MBA2000; Perkin Elmer, Inc., San Jose, CA, USA), and then sequenced with an automated laser fluorescence sequencer (ABI PRISM 3100 Genetic Analyzer; Applied Biosystems; Thermo Fisher Scientific, Inc.). Ten-fold serial dilutions of a known concentration of the product (between 100 and $0.1 \mathrm{pg} / \mu \mathrm{l}$ ) were used to establish the standard curve for qPCR. The qPCR conditions were as follows: 1 cycle for $20 \mathrm{sec}$ at $96^{\circ} \mathrm{C}$, followed by 40 cycles of $2 \mathrm{sec}$ at $96^{\circ} \mathrm{C}$ for denaturation, $15 \mathrm{sec}$ at $60^{\circ} \mathrm{C}$ for annealing, and $15 \mathrm{sec}$ at $72^{\circ} \mathrm{C}$ for extension. The melting program was performed at $72-95^{\circ} \mathrm{C}$ with a heating rate of $1^{\circ} \mathrm{C}$ per $45 \mathrm{sec}$. Spectral data were captured and analyzed using Rotor-Gene Real-Time Analysis Software 6.0 Build 14 (Corbett Life Science; Qiagen, Inc.). All of the samples were run in triplicate. GAPDH was analyzed as an endogenous RNA reference gene and gene expression was normalized to the expression of GAPDH.

Statistical analysis. To develop an easy-to-use risk score, a previously developed strategy using the Cox regression coefficient was adopted for the eight genes in the progression-associated classifier [coagulation factor $\mathrm{C}$ homolog $(\mathrm{COCH})$, EGF LAG seven-pass G-type receptor 3 (CELSR3), heme oxygenase 1 (HMOXI), kinesin family member $1 \mathrm{~A}$ (KIF1A), chromosome 16 open reading frame 74 (MGC17624), methylthioadenosine phosphorylase (MTAP), 6-phosphofructo-2-kinase/fructose-2,6-biphosphatase 4 (PFKFB4) and S100 calcium binding protein A8 (S100A8)] (20). The risk score for each patient was calculated as the sum of each genes score, which was derived by multiplying the expression level of the gene by its corresponding coefficient (Risk score $=\Sigma$ Cox coefficient of gene Gi $x$ expression value of gene $\mathrm{Gi}$ ). Molecular progression risk scores were presented as mean \pm standard deviation and differences across dichotomous categories were assessed using the Mann-Whitney U test or Kruskal-Wallis test. The Kaplan-Meier method was used to estimate time to development of invasive tumor, and differences were assessed using the log-rank test. The
Table II. Baseline characteristics of the patients.

\begin{tabular}{lc}
\hline Parameters & No. of patients, $\mathrm{n}(\%)$ \\
\hline Number of patients & 176 \\
Mean \pm SD age, years (range) & $63.45 \pm 12.72(24-89)$ \\
Median follow-up, months (IQR) & $72.0(37.0-118.7)$ \\
Gender & \\
Male & $146(83.0)$ \\
Female & $30(17.0)$ \\
Tumor size & \\
$\leq 3$ cm & $94(53.4)$ \\
$\geq 3$ cm & $82(46.6)$ \\
Multiplicity & \\
Single & $96(54.5)$ \\
$2-7$ & $56(31.8)$ \\
$\geq 8$ & $24(13.6)$ \\
BCG induction therapy & $126(71.6)$ \\
Stage & \\
Ta & $42(23.9)$ \\
T1 & $134(76.1)$ \\
Grade & \\
1 & \\
3 & $53(30.1)$ \\
Recurrence & \\
Progression to MIBC & \\
& \\
& \\
&
\end{tabular}

$\mathrm{SD}$, standard deviation; IQR, interquartile range; BCG, Bacillus Calmette-Guerin; MIBC, muscle-invasive bladder cancer.

prognostic value of the molecular progression risk score for prediction of development of invasive tumor was analyzed using multivariate Cox proportional hazard regression 
Table III. Progression risk score according to clinicopathological variables.

\begin{tabular}{|c|c|c|c|}
\hline Variables & Number, n (\%) & Molecular progression risk score (mean \pm standard deviation) & P-value \\
\hline Gender & & & $0.159^{\mathrm{a}}$ \\
\hline Male & $146(83.0)$ & $10.82 \pm 2.53$ & \\
\hline Female & $30(17.0)$ & $11.53 \pm 2.36$ & \\
\hline Tumor size & & & $0.199^{\mathrm{a}}$ \\
\hline$\leq 3 \mathrm{~cm}$ & $94(53.4)$ & $10.72 \pm 2.53$ & \\
\hline$\geq 3 \mathrm{~cm}$ & $82(46.6)$ & $11.21 \pm 2.47$ & \\
\hline Multiplicity & & & $0.001^{\mathrm{b}}$ \\
\hline Single & $96(54.5)$ & $10.34 \pm 2.48$ & \\
\hline $2-7$ & $56(31.8)$ & $11.22 \pm 2.23$ & \\
\hline$\geq 8$ & $24(13.6)$ & $12.22 \pm 2.57$ & \\
\hline Stage & & & $0.025^{\mathrm{a}}$ \\
\hline $\mathrm{Ta}$ & $42(23.9)$ & $10.20 \pm 2.76$ & \\
\hline $\mathrm{T} 1$ & $134(76.1)$ & $11.18 \pm 2.38$ & \\
\hline Grade & & & $<0.001^{\mathrm{b}}$ \\
\hline 1 & $53(30.1)$ & $10.09 \pm 2.64$ & \\
\hline 2 & $93(52.8)$ & $10.91 \pm 2.48$ & \\
\hline 3 & $30(17.1)$ & $12.55 \pm 1.34$ & \\
\hline EORTC progression risk score & & & $0.001^{\mathrm{b}}$ \\
\hline 0 & $18(10.2)$ & $9.02 \pm 2.00$ & \\
\hline $2-6$ & $53(30.1)$ & $10.65 \pm 2.79$ & \\
\hline $7-13$ & $100(56.8)$ & $11.41 \pm 2.32$ & \\
\hline $14-23$ & $5(2.8)$ & $11.73 \pm 0.39$ & \\
\hline CUETO progression risk score ${ }^{\mathrm{c}}$ & & & $<0.001^{\mathrm{b}}$ \\
\hline $0-4$ & $68(38.6)$ & $10.21 \pm 2.14$ & \\
\hline $5-6$ & $27(15.3)$ & $10.42 \pm 2.47$ & \\
\hline $7-9$ & $18(10.2)$ & $11.83 \pm 1.70$ & \\
\hline $10-14$ & $13(7.4)$ & $12.91 \pm 1.46$ & \\
\hline Recurrence & & & $0.009^{\mathrm{a}}$ \\
\hline No & $100(56.8)$ & $10.52 \pm 2.48$ & \\
\hline Yes & $76(43.2)$ & $11.51 \pm 2.44$ & \\
\hline Progression to MIBC & & & $<0.001^{\mathrm{a}}$ \\
\hline No & $150(85.2)$ & $10.57 \pm 2.41$ & \\
\hline Yes & $26(14.8)$ & $13.11 \pm 1.87$ & \\
\hline
\end{tabular}

EORTC, European Organization for Research and Treatment of Cancer; CUETO, Spanish Urological Club for Oncological Treatment; MIBC, muscle-invasive bladder cancer. P-value was based on the ${ }^{\mathrm{a}} \mathrm{Mann}-$ Whitney U test or ${ }^{\mathrm{b}}$ Kruskal-Wallis test. ${ }^{\mathrm{c}}$ The CUETO progression risk score category included only data from patients treated with BCG.

models. The molecular progression risk score was estimated as either a continuous or categorical variable, in which classified as having a good-prognosis or poor-prognosis signature, with the 50th percentile (median) of the progression risk score in regression analysis. $\mathrm{P}<0.05$ was considered to indicate a statistically significant difference, and all reported P-values are two-sided. Analyses were performed using the SPSS 21.0 software (IBM SPSS, Armonk, NY, USA).

\section{Results}

Table II summarizes the baseline characteristics of the 176 primary patients with NMIBC included in the analysis. Median follow-up subsequent to surgery was 72.8 months (interquartile range, 37.0-118.7), 30 months longer than in the original study (20). The majority of patients (76.1\%) had stage T1 tumors, and the histological grade distribution was $30.1 \%$ for grade $1,52.8 \%$ for grade 2 and $17.1 \%$ for grade 3 . Of the 176 patients, 73 (43.2\%) experienced recurrence, and 26 (14.8\%) progressed to MIBC.

Molecular progression risk scores were calculated for each patient (Risk score $=\Sigma$ Cox coefficient of gene Gi x expression value of gene $\mathrm{Gi}$, as determined by RT-qPCR). To determine whether molecular grade represented the tumor aggressiveness phenotype, the association between the molecular progression risk score and clinicopathological variables was evaluated. Patients with higher grade, T1 stage or multiple tumors had an increased molecular progression risk score compared with those with lower grade, Ta stage or a single tumor $(\mathrm{P}<0.05$ for all comparisons). Higher progression risk groups according to the 
Table IV. Multivariate Cox regression models for the risk of development of invasive tumors in primary non-muscle-invasive bladder cancer.

\begin{tabular}{|c|c|c|c|c|}
\hline \multirow[b]{2}{*}{ Parameter } & \multicolumn{2}{|c|}{$\begin{array}{l}\text { Multivariate analysis as a } \\
\text { continuous variable }\end{array}$} & \multicolumn{2}{|c|}{$\begin{array}{l}\text { Multivariate analysis as a } \\
\text { categorical variable }\end{array}$} \\
\hline & HR $(95 \% \mathrm{CI})$ & P-value & HR $(95 \% \mathrm{CI})$ & P-value \\
\hline Age (continuous) & $1.031(0.991-1.072)$ & 0.136 & $1.033(0.995-1.072)$ & 0.094 \\
\hline Gender (female) & $0.634(0.205-1.962)$ & 0.429 & $0.822(0.271-2.487)$ & 0.728 \\
\hline Size $(>3 \mathrm{~cm})$ & $2.178(0.889-5.336)$ & 0.088 & $2.371(0.948-5.930)$ & 0.065 \\
\hline \multicolumn{5}{|l|}{ Multiplicity (multiple) } \\
\hline Single & - & & - & \\
\hline $2-7$ & $1.265(0.496-3.224)$ & 0.622 & $1.229(0.497-3.037)$ & 0.655 \\
\hline$\geq 8$ & $1.138(0.368-3.516)$ & 0.823 & $1.493(0.487-4.577)$ & 0.483 \\
\hline \multicolumn{5}{|l|}{ Grade } \\
\hline 1 & - & & - & \\
\hline 2 & $2.182(0.435-10.948)$ & 0.343 & $1.918(0.474-7.756)$ & 0.361 \\
\hline 3 & $2.731(0.454-16.428)$ & 0.272 & $2.663(0.521-13.601)$ & 0.239 \\
\hline Stage (T1) & $1.873(0.369-9.504)$ & 0.449 & $2.116(0.504-8.877)$ & 0.306 \\
\hline Progression risk score (continuous) & $1.489(1.216-1.823)$ & $<0.001$ & - & \\
\hline Progression risk score (high) & - & & $5.026(1.619-15.608)$ & 0.005 \\
\hline
\end{tabular}

HR, hazard ratio; CI, confidence interval.

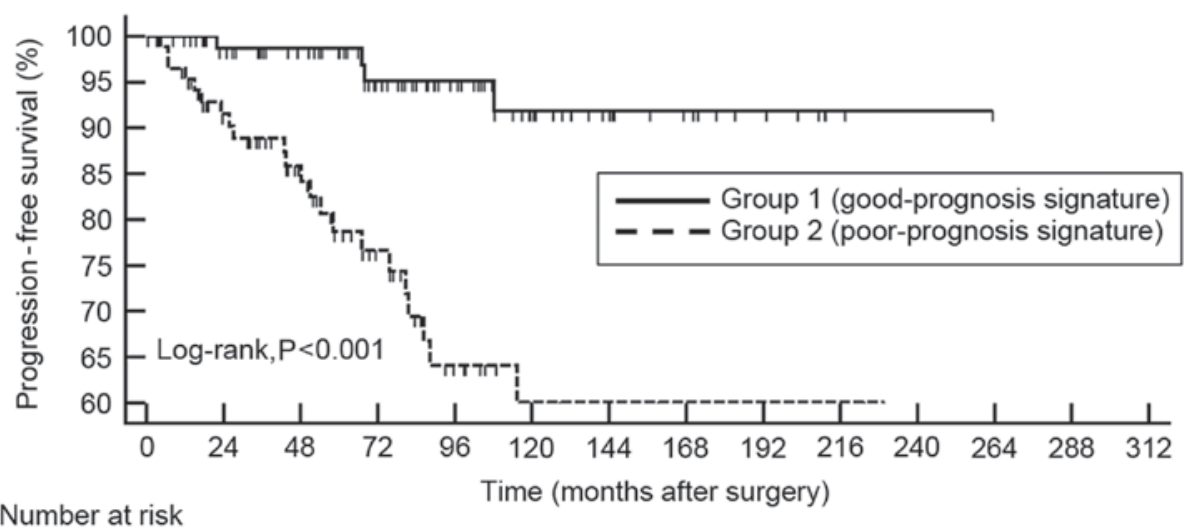

$\begin{array}{lllllllllllll}\text { Group: } 1 & & & & & & & & & & & & \\ \text { Group: } 2 & 88 & 75 & 66 & 49 & 36 & 23 & 16 & 11 & 7 & 2 & 1 & 0 \\ & 88 & 68 & 52 & 34 & 22 & 13 & 8 & 5 & 3 & 2 & 0 & 0\end{array}$

Figure 1. Kaplan-Meier estimation of development of muscle-invasive tumors in primary non-muscle-invasive bladder cancer, according to a molecular progression risk score based on quantitative polymerase chain reaction.

EORTC or CUETO risk tables tended toward higher molecular progression risk scores (Table III).

Kaplan-Meier survival curves revealed significant differences in time to development of MIBC between the good-prognosis and poor-prognosis signature groups (log-rank, $\mathrm{P}<0.001$; Fig. 1). Multivariate Cox regression analysis demonstrated that molecular progression risk score was an independent predictor of tumor invasion, either as a continuous variable [hazard ratio (HR), 1.489; $95 \%$ confidence interval (CI), 1.216-1.823; $\mathrm{P}<0.001]$ or as a categorical variable (HR, 5.026; 95\% CI, 1.619-15.608; P=0.005; Table IV).

\section{Discussion}

The present study aimed to obtain long-term validation of a previously reported progression-associated gene classifier in the prediction of muscle-invasive disease. The present findings revealed that the molecular progression risk score, based on an eight-gene progression-associated classifier, represented biological aggressiveness in the absence of clinical information. Furthermore, the molecular progression risk score was an independent predictor of development of muscular invasion. The molecular progression risk score 
may aid in patient counseling and selection of optimal treatments.

In our previous study, a clinically applicable qPCR gene signature was developed to predict progression of NMIBC $(20,24)$. Our original study identified a progression-associated classifier in patients with NMIBC, including the following eight genes: COCH, CELSR3, HMOX1, KIF1A, $M G C 17624, M T A P, P F K F B 4$ and S100A8. This eight-gene signature was successfully validated in the original and independent cohorts. No cancer progressed in any patients in the good-prognosis signature group (20).

The original study by Kim et al (20) was a comprehensive attempt to identify genetic signatures associated with disease prognosis in $\mathrm{BC}$. By contrast, the present study focused on identifying a molecular predictor of muscle invasion in NMIBC over an extended follow-up period. During the follow-up period, $14.8 \%$ of patients progressed to muscle invasion; therefore, there is a demonstrable requirement for extended follow-up validation. In addition, the original study was unable to conduct multivariate analysis, since no patients with NMIBC in the good-prognosis signature group experienced cancer progression. In the present study, these molecular signatures were validated by multivariate Cox regression analysis and the independent value of the molecular progression risk score in predicting development of tumor invasion was confirmed. In a previous international validation study by Dyrskjøt et al (10), a four-gene classifier was used to predict disease recurrence and progression. Combined analysis with an 88-gene progression classifier and a 68-gene CIS signature yielded a strong hazard ratio of 4.6 in a multivariate Cox regression analysis for prediction of muscle-invasive disease. The main strength of the molecular progression risk score is that it was calculated from the expression levels of only eight genes, making it cost-effective. Another advantage of the progression risk score is that it may be employed not only as a categorical model, but also as a continuous risk score model. To the best of our knowledge, no previous study has reported an independent molecular predictor of muscle invasion in NMIBC that may be employed as a continuous risk score.

It was also investigated whether the molecular progression risk score represented tumor aggressiveness. Furthermore, a significant concordance between pathological aggressive phenotype and molecular progression risk score was observed, and patients with aggressive bladder tumors exhibited increased molecular progression risk scores. Notably, the molecular progression risk score was significantly associated with clinicopathological multivariate progression models, including the EORTC and CUETO risk tables $(13,14)$. Thus, a molecular progression risk score based on gene expression may represent biological aggressiveness, even in the absence of clinical information.

One possible limitation of the present study is that data obtained from a previous study population was used, without adding new cases. However, the present study sought to validate the previously established progression-associated classifier with extended follow-up and a different study design. External validation or collaborative studies are required to confirm the clinical utility of the molecular progression risk score in predicting muscle invasion in NMIBC. Another concern is that BCG maintenance therapy was not considered, since only a small percentage of patients were able to complete the maintenance schedule due largely to BCG-associated side effects. In addition, patients diagnosed with a concomitant CIS, which frequently resembles muscle-invasive disease due to its aggressive biological features, were also excluded from the study. Consequently, the present results may not be reflective of all patients with NMIBC, particularly those with high-risk tumors. Notwithstanding these limitations, the present study represents an important step toward the clinical use of the molecular progression risk score in NMIBC. Introduction of the present molecular progression risk score into routine clinical practice will require additional external validation in a prospective study using a larger number of samples.

In conclusion, the present results identified a molecular progression risk score, based on RT-qPCR analysis, which may represent biological aggressiveness in the absence of clinical information. In the clinic, this progression-associated gene classifier may be used to guide selection of treatment regimen for patients initially diagnosed with NMIBC. Frequent cystoscopic follow-up, adjuvant intravesical instillations or early timing of radical treatment are recommended in patients with a molecular signature associated with poor prognosis.

\section{Acknowledgements}

The present study was supported by the Functional Districts of the Science Belt support program, and the National Research Foundation of Korea (grant nos. NRF-2014R1A2A1A09006983 and NRF-2015R1A2A2A03004100) of Ministry of Science, ICT and Future Planning. The authors wish to thank Ms. Eun-Ju Shim from the National Biobank of Korea at Chungbuk National University Hospital for sample preparation and technical assistance. This work was supported by a research grant from Chungbuk National University, 2012. The specimens for the present study were provided by Chungbuk National University Hospital, a member of the National Biobank of Korea, which is supported by the Ministry of Health, Welfare and Family Affairs.

\section{References}

1. Kim WJ, Park S and Kim YJ: Biomarkers in bladder cancer: Present status and perspectives. Biomark Insights 2: 95-105, 2007.

2. Gregg JR, Dahm P and Chang SS: Guideline-based management of non-muscle invasive bladder cancer. Indian J Urol 31: 320-326, 2015.

3. Clark P, Agarwal N and Biagioli M: NCCN clinical practice guideline in oncology: Bladder cancer. Version I. 2013. Available at NCCN.org. Accessed January 10, 2013.

4. Ahn JH, Jung SI, Yim SU, Kim SW, Hwang EC and Kwon DD: Impact of glycemic control and metformin use on the recurrence and progression of non-muscle invasive bladder cancer in patients with diabetes mellitus. J Korean Med Sci 31: 1464-1471, 2016.

5. Yun SJ, Kim SK and Kim WJ: How do we manage high-grade T1 bladder cancer? Conservative or aggressive therapy? Investig Clin Urol 57 (Suppl 1): S44-S51, 2016.

6. Milowsky MI, Rumble RB, Booth CM, Gilligan T, Eapen LJ, Hauke RJ, Boumansour P and Lee CT: Guideline on muscle-invasive and metastatic bladder cancer (European Association of Urology guideline): American society of clinical oncology clinical practice guideline endorsement. J Clin Oncol 34: 1945-1952, 2016.

7. Witjes JA, Compérat E, Cowan NC, De Santis M, Gakis G, Lebret T, Ribal MJ, Van der Heijden AG and Sherif A; European Association of Urology: EAU guidelines on muscle-invasive and metastatic bladder cancer: Summary of the 2013 guidelines. Eur Urol 65: 778-792, 2014. 
8. Epstein JI, Amin MB, Reuter VR and Mostofi FK: The World Health Organization/International Society of Urological Pathology consensus classification of urothelial (transitional cell) neoplasms of the urinary bladder. Am J Surg Pathol 22: 1435-1448, 1998.

9. May M, Brookman-Amissah S, Roigas J, Hartmann A, Störkel S, Kristiansen G, Gilfrich C, Borchardt R, Hoschke B, Kaufmann O and Gunia S: Prognostic accuracy of individual uropathologists in noninvasive urinary bladder carcinoma: A multicentre study comparing the 1973 and 2004 World Health Organisation classifications. Eur Urol 57: 850-858, 2010.

10. Dyrskjøt L, Zieger K, Real FX, Malats N, Carrato A, Hurst C Kotwal S, Knowles M, Malmström PU, de la Torre M, et al: Gene expression signatures predict outcome in non-muscle-invasive bladder carcinoma: A multicenter validation study. Clin Cancer Res 13: 3545-3551, 2007

11. van Rhijn BW, Burger M, Lotan Y, Solsona E, Stief CG Sylvester RJ, Witjes JA and Zlotta AR: Recurrence and progression of disease in non-muscle-invasive bladder cancer: From epidemiology to treatment strategy. Eur Urol 56: 430-442, 2009.

12. van den Bosch S and Witjes JA: Long-term cancer-specific survival in patients with high-risk, non-muscle-invasive bladder cancer and tumour progression: A systematic review. Eur Urol 60: 493-500, 2011.

13. Sylvester RJ, van der Meijden AP, Oosterlinck W, Witjes JA, Bouffioux C, Denis L, Newling DW and Kurth K: Predicting recurrence and progression in individual patients with stage Ta T1 bladder cancer using EORTC risk tables: A combined analysis of 2596 patients from seven EORTC trials. Eur Urol 49: 466-477, 2006

14. Fernandez-Gomez J, Madero R, Solsona E, Unda M, Martinez-Piñeiro L, Gonzalez M, Portillo J, Ojea A, Pertusa C, Rodriguez-Molina J, et al: Predicting nonmuscle invasive bladder cancer recurrence and progression in patients treated with bacillus Calmette-Guerin: The CUETO scoring model. J Urol 182: 2195-2203, 2009.
15. Kim WJ and Bae SC: Molecular biomarkers in urothelial bladder cancer. Cancer Sci 99: 646-652, 2008.

16. Knowles MA and Hurst CD: Molecular biology of bladder cancer: New insights into pathogenesis and clinical diversity. Nat Rev Cancer 15: 25-41, 2015.

17. Kamat AM, Hegarty PK, Gee JR, Clark PE, Svatek RS, Hegarty N, Shariat SF, Xylinas E, Schmitz-Dräger BJ, Lotan Y, et al: ICUD-EAU international consultation on bladder cancer 2012: Screening, diagnosis, and molecular markers. Eur Urol 63: 4-15, 2013.

18. Kang HW, Yoon HY, Ha YS, Kim WT, Kim YJ, Yun SJ, Lee SC and Kim WJ: FAM70B as a novel prognostic marker for cancer progression and cancer-specific death in muscle-invasive bladder cancer. Korean J Urol 53: 598-606, 2012.

19. Netto GJ: Molecular biomarkers in urothelial carcinoma of the bladder: Are we there yet? Nat Rev Urol 9: 41-51, 2012.

20. Kim WJ, Kim EJ, Kim SK, Kim YJ, Ha YS, Jeong P, Kim MJ, Yun SJ, Lee KM, Moon SK, et al: Predictive value of progression-related gene classifier in primary non-muscle invasive bladder cancer. Mol Cancer 9: 3, 2010.

21. Brausi M, Witjes JA, Lamm D, Persad R, Palou J, Colombel M, Buckley R, Soloway M, Akaza H and Böhle A: A review of current guidelines and best practice recommendations for the management of nonmuscle invasive bladder cancer by the International Bladder Cancer Group. J Urol 186: 2158-2167, 2011.

22. Greene FL: The American Joint Committee on Cancer: Updating the strategies in cancer staging. Bull Am Coll Surg 87: 13-15, 2002.

23. Kirkali Z, Chan T, Manoharan M, Algaba F, Busch C, Cheng L, Kiemeney L, Kriegmair M, Montironi R, Murphy WM, et al: Bladder cancer: Epidemiology, staging, and grading and diagnosis. Urology 66 (6 Suppl 1): S4-S34, 2005.

24. Jeong P, Ha YS, Cho IC, Yun SJ, Yoo ES, Kim IY, Choi YH, Moon SK and Kim WJ: Three-gene signature predicts disease progression of non-muscle invasive bladder cancer. Oncol Lett 2: 679-684, 2011 